\title{
Tumor establishment requires tumor autonomous and non-autonomous deregulation of homeostatic feedback control
}

\author{
Sang Ngo ${ }^{*, 1,2}$, Jackson Liang ${ }^{*, 1,3}$, Yu-Han Su${ }^{1}$, \& Lucy Erin O’Brien ${ }^{1,4}$ \\ * These authors contributed equally to this work \\ ${ }^{1}$ Department of Molecular and Cellular Physiology, Stanford University School of Medicine, Stan- \\ ford, California 94305, USA. \\ ${ }^{2}$ Current address: Department of Neurology, University of California San Francisco, San Francisco, \\ California 94158, USA \\ ${ }^{3}$ Current address: Department of Discovery Oncology, Genentech, South San Francisco, California \\ 94080, USA \\ ${ }^{4}$ Corresponding author, lead contact (email: lucye@stanford.edu)
}

\section{Summary}

In healthy adult organs, robust feedback mechanisms control cell turnover to enforce homeostatic equilibrium between cell division and death [1, 2]. Nascent tumors must subvert these mechanisms to achieve cancerous overgrowth [3-7]. Elucidating the nature of this subversion can reveal how cancers become established and may suggest strategies to prevent tumor progression. In adult Drosophila intestine, a well-studied model of homeostatic cell turnover, the linchpin of cell equilibrium is feedback control of the EGF protease Rhomboid (Rho). Expression of Rho in apoptotic cells enables them to secrete EGFs, which stimulate nearby stem cells to undergo replacement divisions [8]. As in mammals, loss of adenomatous polyposis coli (APC) causes Drosophila intestinal stem cells to form adenomas [9]. Here we demonstrate that Drosophila APC ${ }^{-/}$tumors trigger widespread Rho expression in non-apoptotic cells, resulting in chronic EGF signaling. Initially, nascent $A P C^{-/}$tumors induce rho in neighbor wild-type cells via acute, non-autonomous activation of JNK. During later growth and multilayering, $A P C^{-/-}$ tumors induce rho in tumor cells by autonomous downregulation of E-cadherin (E-cad) and consequent activity of p120-catenin. This sequential dysregulation of tumor nonautonomous and -autonomous EGF signaling converts tissue-level feedback into feedforward activation that drives cancerous overgrowth. Since Rho, EGFR, and E-cad are 
associated with colorectal cancer in humans [10-17], our findings may shed light on how human colorectal tumors progress.

\section{Keywords}

Drosophila, homeostasis, tumor, tumorigenesis, rhomboid, midgut, stem cell, JNK, apoptosis, E-cadherin

\section{Results}

To investigate how tumors subvert cell equilibrium in the Drosophila intestine (midgut) (Figure S1A), we used low-frequency, hs-flp-mediated MARCM recombination [18] to generate stem cells that were (1) marked by heritable fluorescent protein expression and (2) either control genotype or homozygous for null alleles of Drosophila Apc1 and $A p c 2$ (hereafter, $A P C^{-1}$ ) $[19,20]$ (Figure S2A). We allowed these marked stem cells to form multicellular clones and examined clone size and morphology (c.f. STAR Methods, "Clone visualization and quantification"). Midguts from mated females were used exclusively here and in subsequent experiments.

As described previously [21-25], $A P C^{-/}$stem cells frequently gave rise to large, multilayered adenomas over time (Figures 1A, S1B-F). Whereas most 21-day control clones contained fewer than 50 cells, many 21 -day $A P C^{-/}$clones contained 100-500 cells (Figure 1G). This tumorous growth was accompanied by epithelial multilayering. At two days after induction, nearly all $A P C^{-/}$clones were single-layered (Figure S1F); by 21 days after induction, in contrast, $36.2 \pm 1.7 \%$ of $A P C^{-/}$clones were multilayered. These overgrown, multilayered $A P C^{-/}$masses protruded conspicuously into the midgut lumen (Figures S1C, S1D, S1E, S1G), reminiscent of $A P C$-inactivated colonic adenomas in humans [26].

As human $A P C$ cancers progress, they often lose expression of $\mathrm{E}$-cadherin (Ecad, also shotgun) $[27,28]$. We therefore examined whether Drosophila midgut $A P C^{-1-}$ tumors lose E-cad as they develop. In control midguts, E-cad::mTomato [29] localized 
prominently to lateral cell membranes, as expected (Figure 1B, S1E) [8]. In singlelayered $A P C^{-/}$clones and in the basal layers of multilayered $A P C^{-/}$clones, E-cad::mTomato was still present. However in $75 \%$ of supra-basal layers E-cad::mTomato was not detected (Figures 1B, 1C, S1E; see Table S1 for all experimental genotypes). Thus, Drosophila $A P C^{-/}$tumors, like their human counterparts, downregulate E-cad as they progress.

Since human E-cad is an epithelial tumor suppressor, we wondered whether forced E-cad expression would suppress tumor formation. Hence, we assessed the tumorigenicity of $A P C^{-/}$stem cells that ectopically overexpressed E-cad (Figures 1D, S2D). E-cad overexpression had no effect on the sizes of control clones, but markedly reduced the sizes of $A P C^{-/}$clones (Figures 1F, 1G; see Tables S2, S3 for clone statistics). Furthermore, E-cad overexpression sharply reduced the frequency of $A P C^{-/}$ multilayering, from $41.4 \pm 6.3 \%$ of $A P C^{-/}$clones to only $7.1 \pm 3.1 \%$ of $E$-cad-expressing $A P C^{-/}$clones (Figure $1 \mathrm{H}$ ). Thus, E-cad acts as a tumor suppressor in the Drosophila midgut.

We sought to determine how E-cad suppresses midgut tumorigenesis. One model posits that loss of E-cad weakens cell-cell adhesion, facilitating cell invasion that is characteristic of advanced tumor stages. To test whether tumor suppression by E-cad involves adhesion, we forced $A P C^{-/}$stem cells to overexpress an adhesion-incompetent mutant that lacks extracellular adhesion motifs, E-cad ${ }^{d C R 4 h}$ (Figure 1E) [30]. E-cad ${ }^{d C R 4 h}$ substantially prevented $A P C^{-/}$clone overgrowth and multilayering (Figures $1 \mathrm{~F}-\mathrm{H}$ ). Ecad $^{\mathrm{dCR} 4 \mathrm{~h}}$ overexpression did not alter control clone sizes (Figure 1G). These striking results demonstrate that tumor suppression by Drosophila E-cad does not require cadherin-mediated extracellular adhesion.

E-cad's intracellular domain associates with two catenin-family transcription factors, $\beta$-catenin (Armadillo) and p120-catenin (p120) [31]. Although $\beta$-catenin contributes to $A P C$-driven tumorigenesis in both Drosophila midgut and mammalian intestine [21, $22,32,33$, it associates with both tumor suppressive alleles $\left(E-\right.$ cad $^{W T}$ and $E$-cad $\left.{ }^{d C R 4 h}\right)$ and the allele $\mathrm{E}-\mathrm{cad}^{\Delta \mathrm{JM}}$, which we show below is non-suppressive (Figure 1E). Thus, Ecad's ability to suppress tumor growth cannot be attributed to $\beta$-catenin sequestration. 
We next examined $\mathrm{p} 120$. We previously found that, during steady-state turnover [8], E-cad prevents p120 from activating transcription of the EGF protease rhomboid (Figure S1A), likely by sequestering 120 at the enterocyte cortex. We thus examined whether E-cad suppresses tumorigenesis by binding $\mathrm{p} 120$. We forced $A P C^{-/}$stem cells to express $\mathrm{E}$-cad ${ }^{\Delta \mathrm{JM}}$, a mutant with a juxtamembrane deletion that abrogates $\mathrm{p} 120$ but not $\beta$-catenin binding (Figure 1E) [34]. Unlike E-cad and Ecad ${ }^{\text {dCR4h }}$, E-cad ${ }^{\Delta J M}$ failed to suppress tumorigenesis. $A P C^{-/}$clones overexpressing $E$-cad ${ }^{\triangle J M}$ grew to sizes comparable to $A P C^{-/}$clones, and a similar proportion became multilayered (Figures $1 \mathrm{G}, 1 \mathrm{H}$ ). $\mathrm{E}-\mathrm{cad}^{\Delta \mathrm{JM}}$ overexpression did not alter the sizes of control clones (Figure 1G). These results imply that E-cad-p120 binding is crucial for tumor suppression.

If E-cad sequesters $\mathrm{p} 120$ to suppress tumorigenesis, loss of $\mathrm{p} 120$ should also suppress tumorigenesis. To test this prediction, we depleted $p 120$ from $A P C^{-/}$stem cells using RNAi. With $p 120$ depletion, $A P C^{-/}$clones accumulated significantly fewer cells compared to $A P C^{-/}$clones (Figures $1 \mathrm{~F}, 1 \mathrm{G}$ ). They also exhibited less multilayering (Figure 1H). p120 RNAi did not affect control clone sizes (Figure 1G). These findings, combined with the loss of tumor suppression by $E$-cad ${ }^{\Delta J M}$, imply that downregulation of E-cad promotes tumorigenesis by dysregulating $\mathrm{p} 120$.

The key function of p120 during steady-state turnover is to activate rhomboid [8]. We therefore wondered whether p120 contributes to $A P C^{-/}$tumor development by rhomboid activation. First, we examined expression of a rhomboid-lacZ reporter (rholacZ; Figure 2A), which we built into a genetic system for generating negatively marked $A P C^{-/}$clones (Figure S2B) [24]. In this system, all cells initially express GFP, and Flp/FRT recombination generates $A P C^{-/}$stem cells that are unlabeled. Importantly, all cells possessed and were capable of expressing the rhomboid-lacZ transgene.

We observed widespread expression of rhomboid-lacZ in midguts containing $A P C^{-/}$tumors. After 21 days of clone development, rhomboid-lac $Z$ was expressed by $15.3 \pm 12.4 \%$ of cells in $A P C^{-/}$clones but only $1.7 \pm 3.4 \%$ of cells in control clones (Figures $2 \mathrm{~A}-\mathrm{C})$. Furthermore, in midguts that contained $A P C^{-/}$clones, rhomboid-lac $Z$ was detected in $20.0 \pm 12.7 \%$ of cells outside the clones (henceforth referred to as "nonclone cells"); whereas in midguts with control clones, rhomboid-lacZ was detected in on- 
ly $2.1 \pm 1.7 \%$ of non-clone cells (Figures $2 \mathrm{~B}, 3 \mathrm{~A}$ ). This global upregulation of rhomboidlacZ in tumor-containing guts was accompanied by a pronounced increase in rhomboid mRNA (Figure 4F). Intriguingly, $76 \%$ of rhomboid-expressing non-clone cells localized within $\sim 2$ enterocyte diameters $(\sim 30 \mu \mathrm{m})$ of $A P C^{-/}$clones (Figures S4A, S4B). Increased rhomboid expression was not merely caused by $A P C^{+/}$heterozygosity of nonclone cells (c.f. Figure S2B) since numbers of rhomboid-lac $Z^{+}$cells in $A P C^{+/-}$midguts and $A P C^{+/+}$midguts were similar (Figure S4E). Thus, as $A P C^{-/}$tumors develop, rhomboid becomes hyper-induced both tumor autonomously and non-autonomously.

Since Rhomboid enables EGF secretion, its hyper-induction should lead to Egfr hyperactivation. Immunostaining for the activated, di-phosphorylated form of the Egfr effector Erk (dpErk) [4, 8, 22, 35], we found that $30.7 \pm 17.0 \%$ of cells in $A P C^{-/}$clones exhibited dpErk, compared to $5.5 \pm 7.1 \%$ of cells in control clones (Figures $2 \mathrm{E}, 2 \mathrm{~F}$ ). Non-clone cells also exhibited dpErk more frequently in midguts with $A P C^{-/}$clones compared to midguts with control clones (Figure 2E). Frequencies of rhomboid induction and Egfr activation were similar in single-layered and multilayered tumors (Figures $\mathrm{S} 1 \mathrm{H}, \mathrm{S} 1 \mathrm{I})$. Overall, these data show Egfr hyperactivation accompanies rhomboid hyperinduction during $A P C^{-/}$tumor formation.

Does elevated Rhomboid-Egfr signaling promote tumor development? We first investigated this possibility by examining whether developing tumors require tumorautonomous rhomboid. We used MARCM to generate GFP-labeled, $A P C^{-/}$stem cells that additionally expressed rhomboid RNAi (Figures S2A, S2D). The clones arising from these rhomboid $\mathrm{RNAi}, A P C^{-/}$stem cells were markedly smaller than those arising from $A P C^{-/}$stem cells (Figures $2 \mathrm{G}, 2 \mathrm{H}$ ). Moreover, the vast majority of rhomboid RNAi, APC ${ }^{\prime}$ clones did not become multilayered (Figure 2l).

Consistent with this requirement for rhomboid, and similar to prior reports [4, 8, 22, 35-39], depleting egfr blocked both $A P C^{-/}$and control clone growth and $A P C^{-/}$clone multilayering (Figures $2 \mathrm{G}, 2 \mathrm{H}, 2 \mathrm{I}$ ). Thus, hyper-induction of rhomboid in tumors promotes tumorigenesis, likely by potentiating EGFs secretion and consequent Egfr hyperactivation. 
We next assessed whether tumor development requires non-autonomous rhomboid in non-clone cells. We specifically manipulated gene expression in non-clone cells by combining the GeneSwitch system (GSG2326; Figures S2C, S2E) with Flp/FRT recombination to generate $A P C^{-/}$stem cells that lack the GeneSwitch Gal4 driver [24]. In this system, oral administration of RU486 induces UAS-transgene expression specifically in non-clone cells and not $A P C^{-/}$cells. Non-clone cells are distinguished from $A P C^{-/-}$ cells by expression of a recombination-sensitive RFP transgene.

We found that expression of rhomboid RNAi in non-clone cells dramatically reduced $A P C^{-/}$clone sizes (Figure $3 C$ ), such that they approached the sizes of control clones with unmanipulated non-clone cells (Figure 3D, 3E). Multilayering was also substantially reduced (Figure 3F). Overexpressing E-cad or depleting $p 120$ had similar effects (Figures 3D-3F).

The strong growth inhibition that these three non-autonomous manipulations effected on $A P C^{-/}$clones contrasted with their comparatively weak effects on control clones (Figure 3E). This difference implies that tumor non-autonomous E-cad-p120Rhomboid dysregulation specifically fosters tumorigenesis, presumably via tumorautonomous Egfr activation. Consistent with this notion, depleting egfr from non-clone cells did not affect clone sizes or multilayering (Figures 3E-3F).

Together, these results demonstrate that nascent tumors can progress only when the E-cad-p120-Rhomboid pathway is disrupted in both tumors and surrounding, nontumor cells. This dual requirement suggests that both cell populations are needed to produce EGFs in quantities sufficient to overcome robust mechanisms of feedback control.

During normal turnover, rhomboid is suppressed in healthy enterocytes but induced in apoptotic enterocytes (Figure S1A) [8]. This regulatory switch forms the linchpin for tissue-level cell equilibrium by spatiotemporally coupling EGF secretion to the loss of terminally differentiated cells [8]. Given this coupling, we asked whether tumorigenic subversion of cell equilibrium involves deregulation of rhomboid expression. To start, we investigated whether rhomboid-expressing cells were apoptotic by immunostaining against cleaved Caspase3 (c.f. Figure S3A). In control midguts, as ex- 
pected [8], a small minority of rhomboid-lac $Z^{+}$cells was non-apoptotic in both clones (14.5 $\pm 17.0 \%$ ) (Figures 2D, S3B) and non-clone cells (22.5 $\pm 9.8 \%$ ) (Figures 3B, S3B). In midguts with $A P C^{-/}$clones, however, the vast majority of rhomboid-lac $Z^{+}$cells was non-apoptotic in both clones (92.7 $\pm 3.7 \%$ ) (Figures 2D, S3B) and non-clone cells (89.0 $\pm 2.9 \%$ ) (Figures 3B, S3B). This striking finding reveals that the presence of $A P C^{-/}$tumors causes rhomboid to be inappropriately expressed in cells that are not undergoing apoptotic elimination.

Intriguingly, although apoptotic cells comprise a small fraction of the total cell population, Suijkerbuijk et al. previously found that inhibiting apoptosis of non-clone cells reduces the sizes of both $A P C^{-/}$and control clones. We thus probed the role of apoptosis by expressing the potent caspase inhibitor $p 35$ [40] in either $A P C^{-/}$clones (Figures S2A, S2D) or non-clone cells (Figures S2C, S2E). Consistent with prior work [24], we observed that $A P C^{-/}$clones surrounded by p35-expressing non-clone cells were smaller (Figure S3K) [8, 24]. In addition, these clones exhibited reduced levels of Egfr activation and lower frequencies of multilayering compared to $A P C^{-/}$clones with control non-clone cells (Figures S3E-S3F, S3J-S3L). By contrast, $p 35$ expression in $A P C^{-/}$clones had no significant effect on clone sizes, Egfr activation, or multilayering (Figures S3C-S3D, S3G-S3I). Taken together, these results suggest that tumor nonautonomous apoptosis is essential for tumor Egfr activation and growth. This finding is counterintuitive considering only $11 \%$ of rhomboid-expressing non-clone cells are apoptotic (Figure 3B), and the contributing mechanisms are unknown.

How do nascent tumors drive rhomboid hyperinduction? An attractive possibility involves Jun N-terminal kinase (JNK; also basket/bsk). JNK activation is essential for $A P C^{-/}$tumors to grow [24]. Furthermore, JNK-dependent regeneration of damaged midguts is accompanied by widespread induction of rhomboid [38, 41, 42]. Hence, we investigated whether $A P C^{-/}$cells co-opt their neighboring, non-clone cells into expressing rhomboid via JNK. We first examined the kinetics of JNK and rhomboid expression as tumors developed over time. We generated unmarked $A P C^{-/}$stem cells in a background of GFP-labeled non-clone cells (Figure S2B), harvested midguts at 2, 5, 10, or 
21 days after clone induction (Figure 4A), and immunostained non-clone cells for activated, phosphorylated JNK (pJNK) and $\beta$-galactosidase (rho-lacZ; Figures 4B, S4A).

We found that numbers of $\mathrm{JNK}$-activated non-clone cells surged rapidly and dramatically during $A P C^{-/}$tumorigenesis (Figures $4 \mathrm{~B}, 4 \mathrm{C}$ ). pJNK ${ }^{+}$non-clone cells climbed sharply from 2-5 days and remained extremely high from 5-21 days. At 21 days, pJNK ${ }^{+}$non-clone cells were markedly elevated (1151.0 $\pm 190.2 \mathrm{pJNK}^{+}$cells) compared to either wild-type guts with control clones $(10.5 \pm 8.4)$ or genotype-matched $A P C^{+/}$guts in which $A P C^{-/}$clones had not been induced (35.0 \pm 9.1 ) (Figures 4B, S4D). Thus, $A P C^{-/}$clones prompt non-clone cells to acutely hyperactivate JNK early in tumorigenesis, before the mutant clones have become multilayered tumors (Figure S1F).

JNK activation preceded rhomboid induction (Figures 4B-D). After 2 days of $A P C^{-}$ ${ }^{\text {- }}$ clone development, rhomboid-lac $Z^{+}$non-clone cells were still at control levels. After 5 days, they had increased only slightly. From 5-10 days, however, rhomboid-lac $Z^{+}$cells increased dramatically, and from 10-21 days they remained highly elevated. In these tumor-containing guts, $78.3 \pm 7.6 \%$ of non-clone cells that had turned on rhomboid were also pJNK ${ }^{+}$(Figures 4B, 4E, S4A). By contrast, in $A P C^{+/}$guts lacking $A P C^{-/}$clones, only $9.8 \pm 3.2 \%$ of rhomboid-expressing cells were also $\mathrm{pJNK}^{+}$(Figure S4F). Colocalization of rhomboid expression and activated JNK in the same non-clone cells (Figure $4 \mathrm{E}$ ), together with the general delay in rhomboid activation relative to JNK (Figures 4B-4D), raise the possibility that JNK induces rhomboid.

To investigate this possibility, we concomitantly generated $A P C^{-/}$clones and inhibited JNK in non-clone cells via RU486-inducible expression of $b s k^{D N}[43,44]$ (Figures $\mathrm{S} 2 \mathrm{C}, \mathrm{S} 2 \mathrm{E})$. This manipulation reduced rhomboid mRNA by $40 \%$ and diminished Egfractivated tumor cells by $74 \%$ (Figures $4 \mathrm{~F}-4 \mathrm{H}$ ). Furthermore, $A P C^{-/}$clones were substantially smaller, as also observed by Suijkerbuijk et al. [24], and exhibited dramatically less multilayering (Figures S3J-3L).

By comparison, tumor-autonomous expression of $b s k^{D N}$ in $A P C^{-/}$clones did not significantly reduce clone sizes or affect multilayering (Figures S3G-3I), possibly reflect- 
ing that tumor cells do not activate JNK until later stages (Figure 4B). In control midguts, expressing $b s k^{D N}$ in clones or in surrounding non-clone cells did not change clone sizes (Figures S3H, S3K), consistent with prior work [45]. Combined, these kinetic and functional analyses imply a model in which $A P C^{-/}$cells acutely hyperactivate JNK in nonclone cells, which consequently induce rhomboid to activate Egfr and promote tumor growth.

\section{Discussion}

During steady-state turnover of the Drosophila midgut, expression of the EGF protease rhomboid is suppressed in healthy enterocytes and activated in enterocytes undergoing apoptotic elimination [8]. This mechanism provides feedback control so that the mitogenic EGFs Spitz and Keren, both regulated by Rhomboid, become available specifically at the time and place that replacement cells are needed [8]. Here, we have shown that nascent tumors transform this feedback control into feed-forward activation by instigating widespread induction of rhomboid, including in non-apoptotic cells (Figure 4I). This inappropriate rhomboid induction enables EGFs to be secreted chronically, which in turn drives production of new cells regardless of tissue need.

Feedback EGF signaling is transformed into feed-forward activation via sequential non-autonomous and autonomous mechanisms that effectively short-circuit the homeostatic pathway for rhomboid activation. Even before $A P C^{-/}$cells manifest as tumors, they induce rhomboid in wild-type neighbor cells via non-autonomous activation of JNK. During subsequent growth, tumors autonomously activate rhomboid via loss of E-cad and release of p120-catenin in an apoptosis-independent manner. Rhomboid dysregulation in both tumor and non-tumor cells is required to form multilayered adenomas. This dual requirement suggests that high levels of EGFs are necessary to overcome robust enforcement of cell equilibrium.

Suijkerbuijk and colleagues elegantly demonstrated that competition between $A P C^{-/}$cells and wild-type cells leads to tumor growth [24], but the growth-promoting mechanism remained unknown. We suggest cell competition may promote growth by 
267 deregulating rhomboid, which would lead to consequent activation of Egfr. If so, a major 268 implication is that tumor/non-tumor cell competition acts by directly subverting pathways 269 that mediate normal homeostasis. Whether cell competition during mammalian 270 tumorigenesis [46-51] follows a similar template will be important to determine.

The mechanisms that enable establishment of Drosophila $A P C^{-/}$tumors may il272 luminate initiation of human colorectal cancers, which are tightly associated with $A P C$ 273 inactivating mutations. Intriguingly, Rhomboids, E-cad, and EGFR have been implicated 274 in human tumor progression [13, 17, 52-54], suggesting this signaling axis may be conserved. This possibility confers particular interest on two of our findings. First, while loss of E-cad is canonically thought to promote metastasis via loss of cell-cell adhesion, we uncovered a crucial role during early-tumor development: dysregulation of p120-catenin to drive EGF signaling. Whether a similar relationship between p120-catenin and EGF exists in colorectal cancer merits examination. Second, while studies of mammalian Rhomboids have focused on advanced cancers, we find rhomboid induction is a tumorinitiating event. Hence, examining Rhomboids in early-stage mammalian tumorigenesis may be fruitful. Overall, understanding how nascent tumors destabilize cell equilibrium may suggest strategies for preventing potentially tumorigenic cells from establishing tumors. 


\section{Acknowledgements}

S.N. was supported by a Stanford Bio-X Undergraduate Fellowship. J.L. was supported by NSF GRFP DGE-114747 and NIH T32GM007276. This work was supported by ACS RSG-17-167-01-DDC, NIH R01GM116000-01A1, and a Stanford VPUE Faculty Grant to L.E.O. Confocal microscopy was performed at the Stanford Beckman Cell Sciences Imaging Facility (NIH 1S10OD01058001A1). We thank D. Bilder for the gift of cCas-3 antibody; the Developmental Studies Hybridoma Bank for other antibodies; M. Peifer, E. Piddini, M. Fuller, H. Jiang, the Bloomington Drosophila Stock Center (NIH P40OD018537), the TRiP at Harvard Medical School (NIH/NIGMS R01-GM084947), and the Vienna Drosophila Resource Center (http://stockcenter.vdrc.at/control/main) for fly stocks; M. Mirvis, L.J. Koyama, and E.N. Sanders for helpful discussions; J.M. Knapp for writing assistance; and J. Cordero and K. Campbell for comments on the manuscript.

\section{Author Contributions}

J.L., S.N., and L.E.O. designed the study. J.L. and L.E.O. wrote the manuscript. S.N. and Y.H.S. performed genetic crosses, tissue dissection and immunostaining. J.L. designed the experiments, built fly lines, performed QPCR experiments, quantitative analysis of microscopy data, and statistical analysis. S.N., Y.H.S., and J.L. performed confocal microscopy on tissues.

\section{Declaration of Interests}

The authors declare no competing financial interests. 
$310 \quad$ Figure Legends

311

312

Figure 1. Growth of multilayered $A P C^{-/}$tumors requires tumor-autonomous downregulation of E-cadherin (E-cad) and consequent deregulation of p120catenin.

(A) Experimental schema for B and C. Sparsely distributed, GFP-marked stem cells are null for both $A p c$ and $A p c 2\left(A P C^{-/}\right)$. Over the next 21 days, many of these stem cells develop into GFP-marked, multilayered adenomas. See Figures S1B-E and S2A.

(B) Loss of E-cad in supra-basal layers of $A P C^{-/}$tumors. E-cad::mTomato (red hot LUT) is expressed in control tissue (top panels) and in basally localized cells within multilayered $A P C^{-/}$clones (middle panel) but is absent from supra-basal cells (bottom panel). Each image is a single $z$-section of the respective conditions.

(C) Progressive loss of E-cad::mTomato expression in $A P C^{-/}$clones. Percentages of all $A P C^{-/}$clone cells that exhibit E-cad::mTomato are shown for single-layered clones, basal layers of multilayered clones, and supra-basal layers of multilayered clones. $67 \mathrm{sin}$ gle-layered tumors and 38 multilayered $A P C^{-/}$clones pooled from $n=4$ midguts at 21 days post-induction.

(D) Experimental schema for F-H. GFP-marked, $A P C^{-/}$stem cells are generated at 1 day post-eclosion, and gene expression is manipulated specifically within these cells. At 22 days post-eclosion (21 days post-induction), the resulting, GFP-marked stem cell clones are analyzed. See Figures S2A and S2D for genetic strategy.

(E) Domain structure of wild-type (WT) and mutant E-cad alleles. E-cad ${ }^{\text {dCR4h }}$ lacks the extracellular adhesion domain [30]. E-cad ${ }^{\Delta \mathrm{JM}}$ lacks the intracellular binding domain for p120-catenin [34].

(F-H) Ectopic expression of $\mathrm{E}$-cad in nascent $A P C^{-/}$tumors inhibits tumor progression in a p120-dependent, adhesion-independent manner. Images $(\mathbf{F})$ and cell counts $(\mathbf{G})$ of control or $A P C^{-/}$clones with clone-autonomous expression of the indicated transgenes. Cells in clones are marked by GFP. Clone boundaries are outlined in white. In G, $n=3$ midguts per genotype; $p$-values by Mann-Whitney $U$-test. (H) Frequency of multilayered 
338 clones as a percentage of total clones. $n=4$ midguts per genotype. $p$-values by un339 paired $t$-test.

340 For $(\mathbf{C}, \mathbf{G}, \mathbf{H})$, one of three independent experiments is shown with $n$ midguts per exper-

341 iment as indicated. For box-and-whisker plots, boxes show median, 25th and 75th per-

342 centiles, and whiskers are minimum and maximum values. Representative images

343 shown in each panel. All scale bars, $50 \mu \mathrm{m}$. 
Figure 2. Hyperactivation of rhomboid and Egfr in non-apoptotic $A P C^{-/}$cells is essential for tumor growth and multilayering.

(A) Experimental timeline for B-I. Clones are induced one day post-eclosion, and midguts are analyzed at 22 days post-eclosion ( 21 days post-induction).

(B-C) Cells in $\mathrm{APC}^{-/}$clones express rhomboid more frequently than cells in control clones. The genetic schema in Figure S2B was used to generate unmarked clones in background of GFP-expressing 'non-clone' cells (red pseudocolor). Clone boundaries are outlined in white (top and middle rows) and black (bottom row). (B) Immunostaining for a rhomboid-lacZ reporter (rho-lacZ) in midguts with control clones (left column) and $A P C^{-/}$clones (right column). (C) Percentage of cells per clone that express rhomboidlacZ. In the control dataset, 42 clones contain no rhomboid-lac $Z^{+}$cells $(0 \%)$. Clones from $n=3$ midguts per genotype; $P$ values by Mann-Whitney $U$-test.

(D) rhomboid expression in $A P C^{-/}$cells no longer correlates with apoptosis. The genetic schema in Figure S2B was used to generate either control or $A P C^{-/}$clones in midguts with the rhomboid-lacZ reporter. Midguts were immunostained for $\beta$-galactosidase (rhomboid-lacZ) and cleaved Caspase3. Graph shows percentages of $\mathrm{LacZ}^{+}$clone cells that are $\mathrm{Casp3}^{+}$or $\mathrm{Casp3}^{-}$. Whereas most $\mathrm{LacZ}^{+}$cells in control clones are $\mathrm{Casp3}^{+}$, most Lac $Z^{+}$cells in $\mathrm{APC}^{-/}$clones are $\mathrm{Casp3}^{-} . n=4$ midguts per condition. See Figures S3A and S3B for representative images.

(E-F) Cells in $A P C^{-/}$clones activate Erk more frequently than cells in control clones. (E) The genetic schema in Figure S2A was used to generate GFP-marked clones (green, top row). Clone boundaries are outlined in white (top row) and black (bottom row). Erk activation was assessed by immunostaining for di-phosphorylated Erk (dpErk) (top row, red; bottom row, inverted grayscale). (F) Percentages of cells per clone that exhibit dpErk. In the control dataset, 47 clones contain no dpErk ${ }^{+}$cells $(0 \%)$. Clones from $n=3$ midguts per genotype; $p$-values by Mann-Whitney U-test.

(G-I) Tumor growth and multilayering require tumor-autonomous rhomboid and egfr. (G) The genetic schema in Figures S2A and S2D was used to generate GFP-marked clones (green) with clone-autonomous expression of the indicated RNAi transgenes. 
374 Clone boundaries are outlined in white. (H) Sizes of control or $A P C^{-/}$clones that ex-

375 press the indicated transgenes. $n=3$ midguts per genotype; $p$-values by Mann-Whitney

376 U-test. (I) Frequency of multilayered clones as a percentage of total clones. $n=4$

377 midguts per genotype. $p$-values by unpaired $t$-test.

378 For (C-D, F, H-I), one of three independent experiments is shown with $n$ samples as

379 specified for each experiment. For box-and-whisker plots, the boxes show median, $25^{\text {th }}$

380 and $75^{\text {th }}$ percentiles, and whiskers are minimum and maximum values. Representative

381 images shown in each panel. All scale bars, $50 \mu \mathrm{m}$. 
Figure 3. Hyperactivation of rhomboid occurs in non-apoptotic cells surrounding tumors and is essential for tumor growth and multilayering.

(A) Non-clone cells that surround $A P C^{-/}$clones express rhomboid more frequently compared to non-clone cells that surround control clones. Control and $A P C^{-/}$midgut clones were induced using the same experimental protocol and genotypes as Figures 2A-C. Each data point shows the percentage of all non-clone cells that are rhomboid-lac $Z^{+}$in one midgut. Data points in Figures $3 \mathrm{~A}$ and $2 \mathrm{C}$ were obtained from the same midguts. $n$ $=4$ midguts per genotype. $P$ values by unpaired $t$-test.

(B) rhomboid expression in non-clone cells no longer correlates with apoptosis in midguts that contain $A P C^{-/}$clones. The genetic schema in Figure S2B was used to generate either control or $A P C^{-/}$clones in midguts containing rhomboid-lacZ. Midguts were immunostained for rhomboid-lacZ $Z$ and Casp3. Graph shows percentages of all rhomboid-lac $Z^{+}$non-clone cells per midgut that are apoptotic $\left(\mathrm{Casp}^{+}\right)$or non-apoptotic $\left(\right.$ Casp3 $\left.^{-}\right) . n=4$ midguts per genotype. See Figures S3A and S3B for representative images.

(C-F) Tumor growth and multilayering require tumor non-autonomous rhomboid but not egfr. (C) Timeline for generation of clones and concomitant genetic manipulation of nonclone cells. The genetic schema in Figures S2C, 3B was used to generate unmarked clones surrounded by RFP-marked non-clone cells that inducibly express the indicated transgenes upon administration of RU486. All control and experimental animals received RU486 from Day 1 (clone induction) to Day 22 (analysis). (D-E) Images (D) and sizes $(E)$ of control or $A P C^{-/}$clones with non-clone cell expression of the indicated transgenes. Clone boundaries are outlined in white. Scale bar, $50 \mu \mathrm{m}$. $\ln (\mathrm{E}), n=3$ midguts per genotype; $P$ values by Mann-Whitney U-test. (F) Frequency of multilayered clones as a percentage of total clones. $n=4$ midguts per genotype. $P$ values by unpaired $t$-test.

For (A-B, E-F), one of three independent experiments is shown with $n$ numbers as specified for each experiment. For box-and-whisker plots, the boxes show median, $25^{\text {th }}$ and $75^{\text {th }}$ percentiles, and whiskers are minimum and maximum values. Representative images shown in each image panel. 
Figure 4. Nascent $A P C^{-/}$clones induce rhomboid by eliciting JNK activation in surrounding, non-tumor cells.

(A) Experimental timeline for B-E. $A P C^{-/}$clones are induced in animals at 1 day posteclosion and midguts are analyzed 2, 5, 10, and 21 days later.

(B-E) Non-clone cells that surround $A P C^{-/}$clones activate JNK early in tumorigenesis and express rhomboid subsequently. The genetic schema in Figure S2B was used to generate unmarked control or $A P C^{-/}$clones surrounded by GFP-marked non-clone cells in midguts with rhomboid-lacZ. Midguts were immunostained for rhomboid-lacZ and phosphorylated JNK. (B) Representative images of midguts containing either control or $A P C^{-/}$clones at the indicated times after clone induction. Clone boundaries are outlined in white (top two rows) and black (bottom two rows). Top row shows rhomboid-lacZ in green. Second row shows GFP-marked non-clone cells in red. Third row shows pJNK in inverted grayscale in third row. Fourth row shows rhomboid-lac $Z$ in inverted grayscale. Scale bars, $50 \mu \mathrm{m}$. See also Figure S4A. (C, D) Numbers of non-clone cells that are either pJNK ${ }^{+}$(C) or rhomboid-lacZ $Z^{+}$(D) in midguts analyzed at the indicated times. (E) Most non-clone cells that express rhomboid also exhibit JNK activation. Graph shows the percentages of all rhomboid-lacZ ${ }^{+}$non-clone cells that are also $\mathrm{pJNK}^{+}$in midguts analyzed at the indicated times. For (C-E), gray bars represent midguts with control clones and red bars represent midguts with $A P C^{-/}$clones. Each data point represents one midgut. $n=3$ midguts per timepoint; means \pm S.D. One of three independent experiments is shown.

(F-H) JNK activation in non-clone cells promotes rhomboid hyper-induction and tumor cell Erk activation. The genetic strategy in Figures S2C and S2E and experimental protocol in Figure $3 \mathrm{C}$ was used to generate either control or $A P C^{-/-}$clones and concomitantly express a dominant negative allele of JNK ( $\left.b s k^{D N}\right)$ in non-clone cells.

(F) Inhibition of JNK in non-clone cells reduces levels of rhomboid mRNA. Wholemidgut qPCR was performed using midguts of the indicated genotypes. mRNA levels are shown normalized to midguts that contain control clones (left bar). Bars represent means \pm S.D.; three biological replicates per condition. $P$ values by unpaired $t$-test. 
441 (G-H) Inhibition of JNK in non-clone cells reduces Erk activation in $A P C^{-/}$clones.

442 Immunostaining for dpErk was performed on $A P C^{-/-}$clone-containing midguts with or

443 without $b s k^{D N}$ expression in non-clone cells. Percentages of cells per clone that are

$444 \operatorname{dpErk}^{+}(\mathbf{G})$ and representative images $(\mathbf{H})$ are shown. In the $b s k^{D N}$ condition, 35 clones

445 contain zero $\mathrm{dpErk}^{+}$cells (0\%). Clones from $n=3$ midguts per genotype; $P$ values by

446 Mann-Whitney $U$-test. One of three independent experiments is shown. Scale bars, 50

$447 \mu \mathrm{m}$.

448 (I) Model. Tumor establishment requires that tumorigenic stem cells de-stabilize cell

449 equilibrium by coercing non-apoptotic cells to express rhomboid. During healthy turno-

450 ver (left), stem cell division is coupled to enterocyte death because expression of rhom-

451 boid, and hence secretion of mitogenic EGFs, occurs via apoptotic downregulation of E-

452 cad and consequent release of p120-catenin [8]. For new tumors to become established

453 (right), tumor-initiating stem cells decouple division from death by instigating widespread

454 expression of rhomboid in cells that are not apoptotic. rhomboid hyper-induction is ini-

455 tially tumor non-autonomous, via activation of JNK in non-clone cells. It subsequently

456 becomes tumor autonomous, via downregulation of E-cad and consequent activity of

457 p120-catenin in tumor cells. 


\section{STAR Methods}

\section{Lead Contact and Materials Availability}

Further information and requests for resources and reagents should be directed to and will be fulfilled by the Lead Contact, Lucy Erin O'Brien (lucye@stanford.edu). This study did not generate new unique reagents.

\section{Experimental Model and Subject Details}

Adult female flies (Drosophila melanogaster) were used in all experiments. Crosses and adult flies were raised at $25^{\circ} \mathrm{C}$ in vials containing molasses-cornmeal. Unless specified otherwise, flies were heat-shocked 1 day after eclosion to induce clones and collected 21 days after induction for dissection/ immunostaining. See Table S1 for full list of experimental genotypes.

\section{Fly stocks}

The following stocks were obtained from the Bloomington Stock Center: $y$ w shg ${ }^{\text {mTomato }}$, UAS-shg, UAS-shg ${ }^{\triangle J M}$, UAS-egfr RNAi (TRiP.HMS05003), UAS-rho RNAi (TRiP.HMS02264), UAS-bsk ${ }^{D N}$, and UAS-p35. UAS-p120 RNAi (KK113572) was obtained from the Vienna Drosophila Resource Center. The following stocks were generous gifts: FRT82 APC2 ${ }^{G 10} A P C 1^{Q 8}$ (from M. Peifer), hsflp ${ }^{122}$; FRT82 ubi-GFP and hsflp ${ }^{122}$; FRT82 GS2326 ubi-RFP (from E. Piddini [24]), UAS-shg ${ }^{d C R 4 h}$ (from M. Fuller), and $r h 0^{X 81}$ (rhomboid-lacZ, from $\mathrm{H}$. Jiang). Other stocks (from our previous studies [8, 55]): w; FRT82 and w UAS-CD8:GFP hsflp ${ }^{122}$; tubGAL4; FRT82 tubGAL80. Detailed information on Drosophila genes and stocks is available from FlyBase (http://flybase.org/).

\section{Method Details}

Induction of stem cell clones

Tumor clones were generated using three separate labeling systems (Figure S2). For all three labeling systems, tumor clones were generated by collecting adult flies one day post-eclosion and performing two $30-\mathrm{min}, 38.5^{\circ} \mathrm{C}$ heat shocks separated by a 8 -min chill on ice. Flies were returned to $25^{\circ} \mathrm{C}$ until time of dissection. For experiments which manipulated gene expression in adjacent tissue after tumor induction (Figure S2C; also 
known as the "pLoser" system [24]), both control and experimental flies were fed RU486 upon returning to $25^{\circ} \mathrm{C}$ post-heat shock until time of dissection (see "GeneSwitch induction" below).

\section{GeneSwitch induction}

To induce expression of the GeneSwitch driver, GS2326, adult flies (control and experimental cohorts) were fed RU486. RU486 (Sigma-Aldrich) was dissolved in $\mathrm{dH}_{2} \mathrm{O}$ to reach a working concentration of $25 \mu \mathrm{g} / \mathrm{mL}$. This solution was used to prepare yeast paste, which was fed to flies as a supplement to their standard cornmeal-molasses diet for the duration of induced gene expression. Drug-containing yeast paste was replenished every three days.

\section{Bleomycin feeding}

Bleomycin (Sigma-Aldrich) was prepared at a working concentration of $25 \mu \mathrm{g} / \mathrm{ml}$, dissolved in $\mathrm{ddH}_{2} \mathrm{O}$ with $5 \%$ sucrose. This solution was used to prepare yeast paste, which was fed to flies as a supplement to their standard cornmeal-molasses diet for 5 hours.

\section{Immunohistochemistry and microscopy}

Immunohistochemistry samples were prepared by incubating in fixative ( $8 \%$ formaldehyde, $200 \mathrm{mM}$ Na cacodylate, $100 \mathrm{mM}$ sucrose, 40mM KOAc, 10mM NaOAc, and $10 \mathrm{mM}$ EGTA) for $20 \mathrm{~min}$ at room temperature. Fixed issues were immunostained and mounted in agarose (see also [8,55]). Anti-GFP and anti-RFP antibodies were used to improve detection of $u b i-G F P$ and $u b i-R F P$ expression in tumor labeling systems (Figure S2). Anti-RFP was used to detect shg ${ }^{\text {mTomato }}$. Primary antibodies: mouse anti- $\beta$ galactosidase (1:400, Promega Z3781), rabbit anti-cleaved caspase-3 (1:400, Cell Signaling, gift from D. Bilder), rabbit anti-dpErk (1:200, Cell Signaling 4370P), mouse antiCoracle (1:400, DSHB C615.16), mouse anti-Discs large (1:400, DSHB 4F3), rabbit anti-pJNK pTPpY (1:500, Promega V7931), chicken anti-GFP (1:400, Invitrogen A10262) rabbit anti-RFP (1:500, Invitrogen R10367), and mouse anti-RFP (1:500, Invitrogen RF5R). Secondary antibodies: Alexa Fluor 488-, 555- or 647-conjugated anti-rabbit, anti-mouse, or anti-chicken IgGs (1:800, LifeTechnologies A31570, A11001, A11039, A32728, A32732, and A21244). Nuclei were stained with DAPI (LifeTechnologies, 
1:1,000). Samples were mounted in ProLong (LifeTechnologies). Imaging of samples was performed on a Leica SP8 confocal microscope, with serial optical sections taken at $3.5 \mu \mathrm{m}$ intervals through the entirety of whole-mounted, immunostained midguts.

\section{gRT-PCR}

mRNA was extracted from whole midguts using Trizol reagent (Invitrogen); four midguts per biological replicate. Extracted RNA was used for cDNA synthesis with Invitrogen SuperStrand III First Script Super Mix (Invitrogen). Real-time PCR was performed using the relative standard curve method with SYBR GreenER Supermix (Invitrogen) on a StepOnePlus ABI machine. Each biological replicate was assessed in three technical replicate experiments. Expression levels were normalized to non-tumorous midguts expressing control clones; rp49 transcripts were used as a reference. Primers were from $[8,56]$. Primer sequences from 5' to 3' - rp49 Fwd: CGGATCGATATGCTAAGCTGT, rp49 Rev: CGACGCACTCTGTTGTCG, rhomboid Fwd:

GAGCACATCTACATGCAACGC, and rhomboid Rev:

GGAGATCACTAGGATGAACCAGG.

\section{Study design}

Sample sizes were chosen based on our previous studies [8,55], which also characterized changes in clone sizes and midgut cell numbers; see also Table S2. Most experiments were replicated three times; see respective figure legends. No exclusion criteria were applied. No sample randomization or blinding was performed.

\section{Quantification and Statistical Analysis}

Clone visualization and quantification

Tumors were visualized (1) as z-stacks using Fiji [57] and (2) in 3D using the Bitplane Imaris software. For each midgut, all clones within the R4 and R5 regions [58] were analyzed (see Figure S1B). Cells per tumor were measured as the number of $\mathrm{DAPI}^{+}$ nuclei within the labeled clone boundary (as determined by the presence or absence of respective labeling proteins). All clone counts were performed manually. To categorize 
548 clones as single-layered or multilayered, each clone was viewed from the sagittal plane

549 in Bitplane Imaris. Clones were designated as single-layered if all polyploid enterocytes

550 possessed both (1) a free luminal surface that was not juxtaposed to other cells or to

551 presumptive basement membrane/visceral muscle and (2) a basal surface that was not

552 juxtaposed to other cells but was juxtaposed to presumptive basement mem-

553 brane/visceral muscle. Clones were designated as multi-layered if one or more polyploid

554 enterocytes lacked either or both of these criteria. Statistical parameters for clone re-

555 ported in Table S3.

Statistical analysis

All statistical analyses were performed using Graphpad Prism 7. For comparisons of clone size distributions, unpaired two-tailed Mann-Whitney U-tests were used to assess statistical significance. To compare the frequencies of mutilayered clones, cell numbers or percentages, and mRNA levels, unpaired two-tailed $t$-tests were used to assess statistical significance. No methods were applied to test the assumptions for respective statistical approaches. Statistical parameters - e.g. sample sizes $(n)$ and values represented by error bars or boxplots - are reported in respective figure legends; $p$-values are reported in respective graphs. For each experiment, $\mathrm{n}$ represents the number of midguts per condition.

569 This study did not generate or analyze any datasets/code. 


\section{References}

1. Pellettieri, J., and Sanchez Alvarado, A. (2007). Cell turnover and adult tissue homeostasis: from humans to planarians. Annu Rev Genet 41, 83-105.

2. O'Brien, L.E., and Bilder, D. (2013). Beyond the niche: tissue-level coordination of stem cell dynamics. Annu Rev Cell Dev Biol 29, 107-136.

3. Brown, S., Pineda, C.M., Xin, T., Boucher, J., Suozzi, K.C., Park, S., MatteMartone, C., Gonzalez, D.G., Rytlewski, J., Beronja, S., et al. (2017). Correction of aberrant growth preserves tissue homeostasis. Nature 548, 334-337.

4. Patel, P.H., Dutta, D., and Edgar, B.A. (2015). Niche appropriation by Drosophila intestinal stem cell tumours. Nat Cell Biol 17, 1182-1192.

5. Egeblad, M., Nakasone, E.S., and Werb, Z. (2010). Tumors as organs: complex tissues that interface with the entire organism. Dev Cell 18, 884-901.

6. Brock, A., Krause, S., and Ingber, D.E. (2015). Control of cancer formation by intrinsic genetic noise and microenvironmental cues. Nat Rev Cancer 15, 499509.

7. Quail, D.F., and Joyce, J.A. (2013). Microenvironmental regulation of tumor progression and metastasis. Nat Med 19, 1423-1437.

8. $\quad$ Liang, J., Balachandra, S., Ngo, S., and O'Brien, L.E. (2017). Feedback regulation of steady-state epithelial turnover and organ size. Nature 548, 588591.

9. Tian, A., Benchabane, H., and Ahmed, Y. (2018). Wingless/Wnt Signaling in Intestinal Development, Homeostasis, Regeneration and Tumorigenesis: A Drosophila Perspective. J Dev Biol 6.

10. Rodriguez, F.J., Lewis-Tuffin, L.J., and Anastasiadis, P.Z. (2012). E-cadherin's dark side: possible role in tumor progression. Biochim Biophys Acta 1826, 23-31.

11. Brooks, S.A., Lomax-Browne, H.J., Carter, T.M., Kinch, C.E., and Hall, D.M. (2010). Molecular interactions in cancer cell metastasis. Acta Histochem 112, 325.

12. Yu, Y., and Elble, R.C. (2016). Homeostatic Signaling by Cell-Cell Junctions and Its Dysregulation during Cancer Progression. J Clin Med 5.

13. Jeanes, A., Gottardi, C.J., and Yap, A.S. (2008). Cadherins and cancer: how does cadherin dysfunction promote tumor progression? Oncogene 27, 69206929.

14. Roskoski, R. (2014). The ErbB/HER family of protein-tyrosine kinases and cancer. Pharmacol Res 79, 34-74.

15. Yewale, C., Baradia, D., Vhora, I., Patil, S., and Misra, A. (2013). Epidermal growth factor receptor targeting in cancer: a review of trends and strategies. Biomaterials 34, 8690-8707.

16. Cheng, T.L., Lai, C.H., Jiang, S.J., Hung, J.H., Liu, S.K., Chang, B.I., Shi, G.Y., and Wu, H.L. (2014). RHBDL2 is a critical membrane protease for anoikis resistance in human malignant epithelial cells. ScientificWorldJournal 2014, 902987.

17. Song, W., Liu, W., Zhao, H., Li, S., Guan, X., Ying, J., Zhang, Y., Miao, F., Zhang, M., Ren, X., et al. (2015). Rhomboid domain containing 1 promotes 
colorectal cancer growth through activation of the EGFR signalling pathway. Nature Communications 6, 8022.

18. Lee, T., and Luo, L. (1999). Mosaic analysis with a repressible cell marker for studies of gene function in neuronal morphogenesis. Neuron 22, 451-461.

19. Ahmed, Y., Hayashi, S., Levine, A., and Wieschaus, E. (1998). Regulation of armadillo by a Drosophila APC inhibits neuronal apoptosis during retinal development. Cell 93, 1171-1182.

20. McCartney, B.M., Price, M.H., Webb, R.L., Hayden, M.A., Holot, L.M., Zhou, M., Bejsovec, A., and Peifer, M. (2006). Testing hypotheses for the functions of APC family proteins using null and truncation alleles in Drosophila. Development 133, 2407-2418.

21. Lee, W.C., Beebe, K., Sudmeier, L., and Micchelli, C.A. (2009). Adenomatous polyposis coli regulates Drosophila intestinal stem cell proliferation. Development 136, 2255-2264.

22. Wang, C., Zhao, R., Huang, P., Yang, F., Quan, Z., Xu, N., and Xi, R. (2013). APC loss-induced intestinal tumorigenesis in Drosophila: Roles of Ras in Wnt signaling activation and tumor progression. Dev Biol 378, 122-140.

23. Cordero, J.B., Stefanatos, R.K., Myant, K., Vidal, M., and Sansom, O.J. (2012). Non-autonomous crosstalk between the Jak/Stat and Egfr pathways mediates Apc1-driven intestinal stem cell hyperplasia in the Drosophila adult midgut. Development 139, 4524-4535.

24. Suijkerbuijk, S.J., Kolahgar, G., Kucinski, I., and Piddini, E. (2016). Cell Competition Drives the Growth of Intestinal Adenomas in Drosophila. Curr Biol 26, 428-438.

25. Tian, A., Benchabane, H., Wang, Z., Zimmerman, C., Xin, N., Perochon, J., Kalna, G., Sansom, O.J., Cheng, C., Cordero, J.B., et al. (2017). Intestinal stem cell overproliferation resulting from inactivation of the APC tumor suppressor requires the transcription cofactors Earthbound and Erect wing. PLoS Genet 13, e1006870.

26. Fearon, E.R. (2011). Molecular genetics of colorectal cancer. Annu Rev Pathol 6, 479-507.

27. Dorudi, S., Sheffield, J.P., Poulsom, R., Northover, J.M., and Hart, I.R. (1993). Ecadherin expression in colorectal cancer. An immunocytochemical and in situ hybridization study. Am J Pathol 142, 981-986.

28. Hao, X., Palazzo, J.P., Ilyas, M., Tomlinson, I., and Talbot, I.C. (1997). Reduced expression of molecules of the cadherin/catenin complex in the transition from colorectal adenoma to carcinoma. Anticancer research 17, 2241-2247.

29. Huang, J., Zhou, W., Dong, W., Watson, A.M., and Hong, Y. (2009). Directed, efficient, and versatile modifications of the Drosophila genome by genomic engineering. Proc Natl Acad Sci U S A 106, 8284-8289.

30. Oda, H., and Tsukita, S. (1999). Nonchordate classic cadherins have a structurally and functionally unique domain that is absent from chordate classic cadherins. Dev Biol 216, 406-422.

31. McCrea, P.D., and Gottardi, C.J. (2016). Beyond $\beta$-catenin: prospects for a larger catenin network in the nucleus. Nat Rev Mol Cell Biol 17, 55-64. 
32. Huels, D.J., Ridgway, R.A., Radulescu, S., Leushacke, M., Campbell, A.D., Biswas, S., Leedham, S., Serra, S., Chetty, R., Moreaux, G., et al. (2015). Ecadherin can limit the transforming properties of activating $\beta$-catenin mutations. EMBO J 34, 2321-2333.

33. Phelps, R.A., Chidester, S., Dehghanizadeh, S., Phelps, J., Sandoval, I.T., Rai, K., Broadbent, T., Sarkar, S., Burt, R.W., and Jones, D.A. (2009). A two-step model for colon adenoma initiation and progression caused by APC loss. Cell 137, 623-634.

34. Pacquelet, A., and Rørth, P. (2005). Regulatory mechanisms required for DEcadherin function in cell migration and other types of adhesion. J Cell Biol 170, 803-812.

35. Cordero, J.B., Ridgway, R.A., Valeri, N., Nixon, C., Frame, M.C., Muller, W.J., Vidal, M., and Sansom, O.J. (2014). c-Src drives intestinal regeneration and transformation. EMBO J 33, 1474-1491.

36. Biteau, B., and Jasper, H. (2011). EGF signaling regulates the proliferation of intestinal stem cells in Drosophila. Development 138, 1045-1055.

37. Buchon, N., Broderick, N.A., Kuraishi, T., and Lemaitre, B. (2010). Drosophila EGFR pathway coordinates stem cell proliferation and gut remodeling following infection. BMC Biol 8, 152.

38. Jiang, H., Grenley, M.O., Bravo, M.J., Blumhagen, R.Z., and Edgar, B.A. (2011). EGFR/Ras/MAPK signaling mediates adult midgut epithelial homeostasis and regeneration in Drosophila. Cell Stem Cell 8, 84-95.

39. Strand, M., and Micchelli, C.A. (2011). Quiescent gastric stem cells maintain the adult Drosophila stomach. Proc Natl Acad Sci U S A 108, 17696-17701.

40. Hay, B.A., Wolff, T., and Rubin, G.M. (1994). Expression of baculovirus P35 prevents cell death in Drosophila. Development 120, 2121-2129.

41. Jiang, H., Patel, P.H., Kohlmaier, A., Grenley, M.O., McEwen, D.G., and Edgar, B.A. (2009). Cytokine/Jak/Stat signaling mediates regeneration and homeostasis in the Drosophila midgut. Cell 137, 1343-1355.

42. Zhang, P., Holowatyj, A.N., Roy, T., Pronovost, S.M., Marchetti, M., Liu, H., Ulrich, C.M., and Edgar, B.A. (2019). An SH3PX1-Dependent EndocytosisAutophagy Network Restrains Intestinal Stem Cell Proliferation by Counteracting EGFR-ERK Signaling. Dev Cell 49, 574-589.e575.

43. Zhai, Z., Kondo, S., Ha, N., Boquete, J.P., Brunner, M., Ueda, R., and Lemaitre, B. (2015). Accumulation of differentiating intestinal stem cell progenies drives tumorigenesis. Nat Commun 6, 10219.

44. Ma, X., Shao, Y., Zheng, H., Li, M., Li, W., and Xue, L. (2013). Src42A modulates tumor invasion and cell death via Ben/dUev1a-mediated JNK activation in Drosophila. Cell Death Dis 4, e864.

45. Kolahgar, G., Suijkerbuijk, S.J., Kucinski, I., Poirier, E.Z., Mansour, S., Simons, B.D., and Piddini, E. (2015). Cell Competition Modifies Adult Stem Cell and Tissue Population Dynamics in a JAK-STAT-Dependent Manner. Dev Cell 34, 297-309.

46. Madan, E., Pelham, C.J., Nagane, M., Parker, T.M., Canas-Marques, R., Fazio, K., Shaik, K., Yuan, Y., Henriques, V., Galzerano, A., et al. (2019). Flower isoforms promote competitive growth in cancer. Nature 572, 260-264. 
47. Martins, V.C., Busch, K., Juraeva, D., Blum, C., Ludwig, C., Rasche, V., Lasitschka, F., Mastitsky, S.E., Brors, B., Hielscher, T., et al. (2014). Cell competition is a tumour suppressor mechanism in the thymus. Nature 509, 465470.

48. Snippert, H.J., Schepers, A.G., van Es, J.H., Simons, B.D., and Clevers, H. (2014). Biased competition between Lgr5 intestinal stem cells driven by oncogenic mutation induces clonal expansion. EMBO Rep 15, 62-69.

49. Vermeulen, L., Morrissey, E., van der Heijden, M., Nicholson, A.M., Sottoriva, A., Buczacki, S., Kemp, R., Tavaré, S., and Winton, D.J. (2013). Defining stem cell dynamics in models of intestinal tumor initiation. Science 342, 995-998.

50. Baker, N.E., and Li, W. (2008). Cell competition and its possible relation to cancer. Cancer Res 68, 5505-5507.

51. Moreno, E. (2008). Is cell competition relevant to cancer? Nat Rev Cancer 8, 141-147.

52. Lacunza, E., Canzoneri, R., Rabassa, M.E., Zwenger, A., Segal-Eiras, A., Croce, M.V., and Abba, M.C. (2012). RHBDD2: a 5-fluorouracil responsive gene overexpressed in the advanced stages of colorectal cancer. Tumour Biol 33, 2393-2399.

53. Tsanou, E., Peschos, D., Batistatou, A., Charalabopoulos, A., and Charalabopoulos, K. (2008). The E-cadherin adhesion molecule and colorectal cancer. A global literature approach. Anticancer Res 28, 3815-3826.

54. Zhang, M., Miao, F., Huang, R., Liu, W., Zhao, Y., Jiao, T., Lu, Y., Wu, F., Wang, $\mathrm{X}$., Wang, $\mathrm{H}$., et al. (2018). RHBDD1 promotes colorectal cancer metastasis through the Wnt signaling pathway and its downstream target ZEB1. J Exp Clin Cancer Res 37, 22.

55. O'Brien, L.E., Soliman, S.S., Li, X., and Bilder, D. (2011). Altered modes of stem cell division drive adaptive intestinal growth. Cell 147, 603-614.

56. Amcheslavsky, A., Song, W., Li, Q., Nie, Y., Bragatto, I., Ferrandon, D., Perrimon, N., and Ip, Y.T. (2014). Enteroendocrine cells support intestinal stemcell-mediated homeostasis in Drosophila. Cell Rep 9, 32-39.

57. Schindelin, J., Arganda-Carreras, I., Frise, E., Kaynig, V., Longair, M., Pietzsch, T., Preibisch, S., Rueden, C., Saalfeld, S., Schmid, B., et al. (2012). Fiji: an opensource platform for biological-image analysis. Nat Methods 9, 676-682.

58. Buchon, N., Osman, D., David, F.P., Fang, H.Y., Boquete, J.P., Deplancke, B., and Lemaitre, B. (2013). Morphological and molecular characterization of adult midgut compartmentalization in Drosophila. Cell Rep 3, 1725-1738. 
A

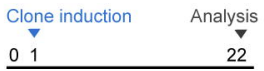

Adult age (days)

rho-lacZ, hsflp, FRT82B ubi-nls:GFP

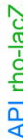

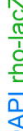
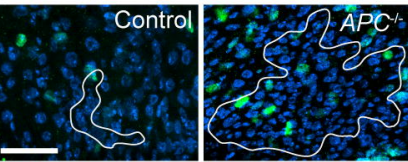

0
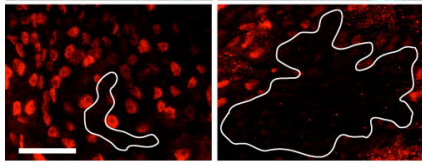

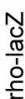

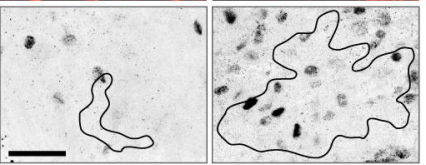

Clones / Non-clone cells (ubi-nls:GFP)

G

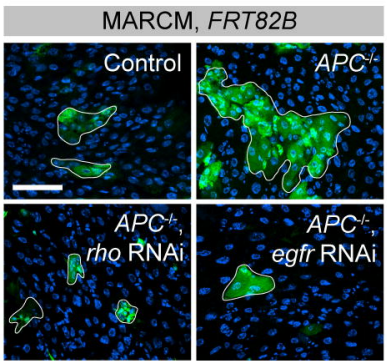

Clones (GFP) / Non-clone cells
C

$60, p<.0001$
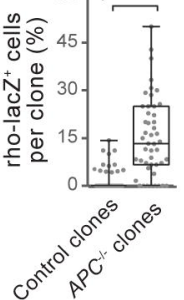

E

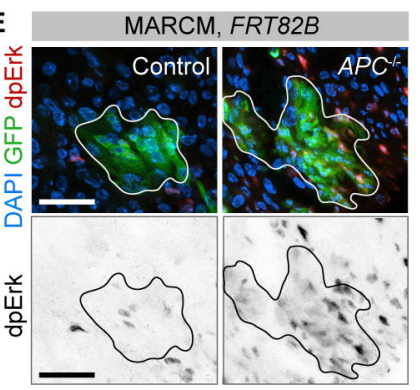

Clones (GFP) / Non-clone cells

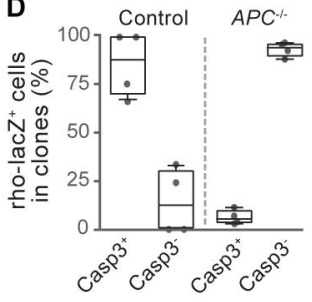

$\mathbf{F}$

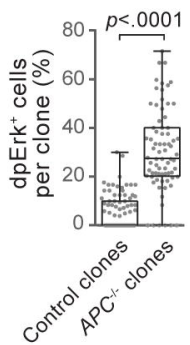

H

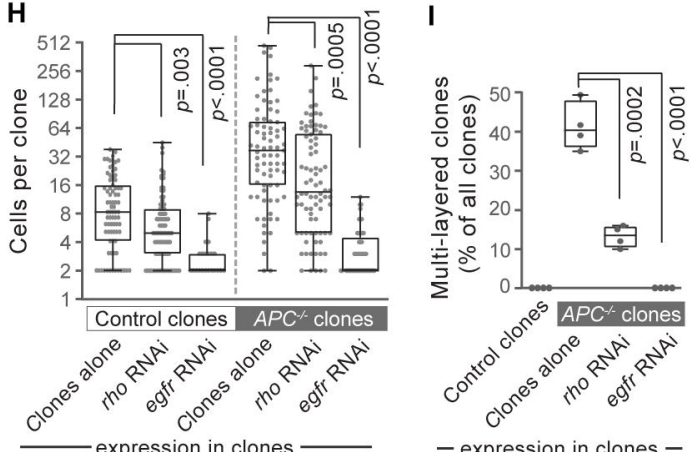




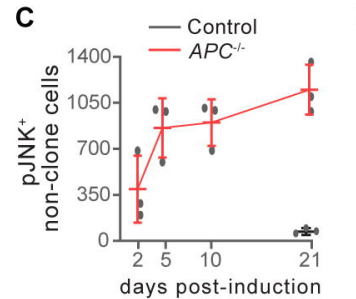

D $\quad$ E

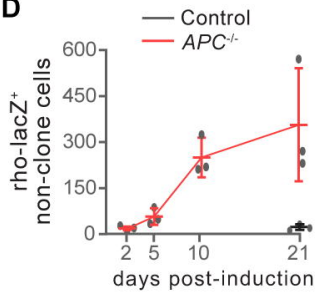

E $\quad$ Control

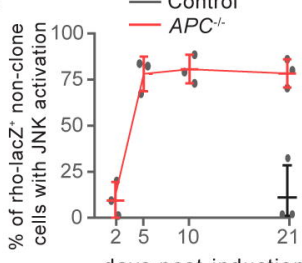

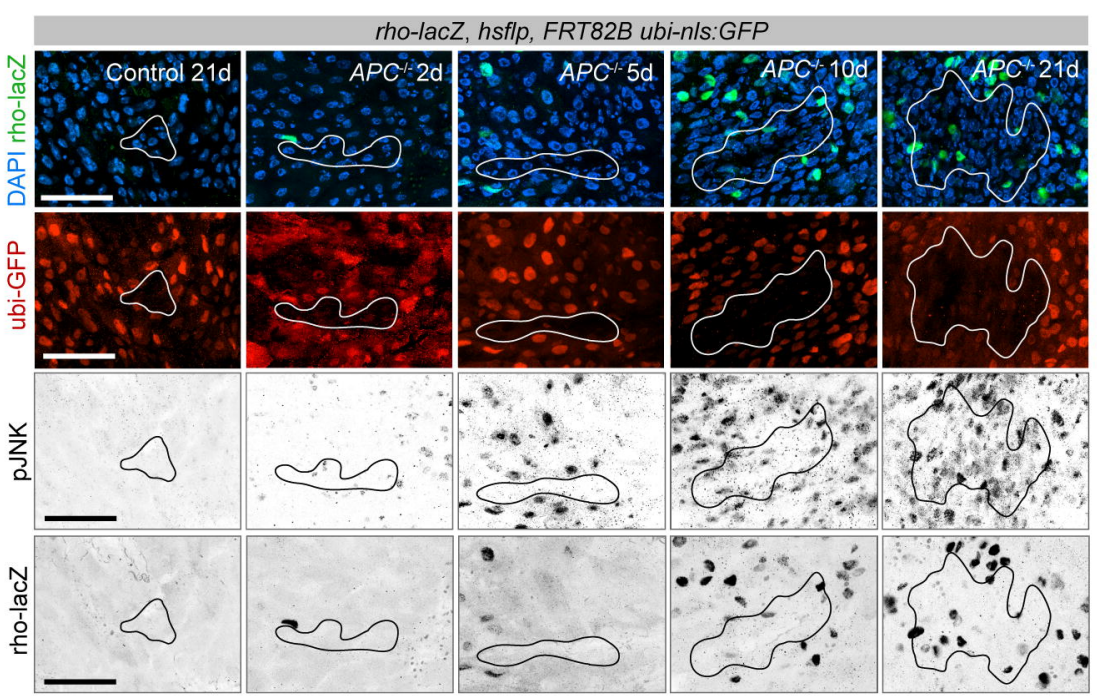

$A P C^{-}$clones / Non-clone cells (ubi-nls:GFP)
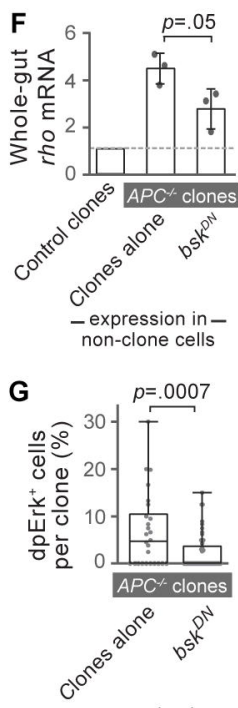

- expression in non-clone cells

Tumor progression

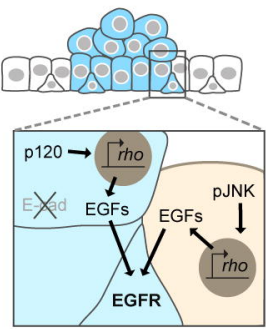

Chronic production of proliferative cues by tumor and non-tumor cells 